\title{
Chitosan-Alginate Nanoparticle System Efficiently Delivers Doxorubicin to MCF-7 Cells
}

\author{
Nuwanthi P. Katuwavila, ${ }^{1,2}$ A. D. L. Chandani Perera, ${ }^{1,3}$ \\ Sameera R. Samarakoon, ${ }^{4}$ Preethi Soysa, ${ }^{5}$ Veranja Karunaratne, ${ }^{2,3}$ \\ Gehan A. J. Amaratunga, ${ }^{2,6}$ and D. Nedra Karunaratne ${ }^{1,3}$ \\ ${ }^{1}$ Postgraduate Institute of Science, University of Peradeniya, 20400 Peradeniya, Sri Lanka \\ ${ }^{2}$ Sri Lanka Institute of Nanotechnology, Mahenwatta, Pitipana, 10200 Homagama, Sri Lanka \\ ${ }^{3}$ Department of Chemistry, Faculty of Science, University of Peradeniya, 20400 Peradeniya, Sri Lanka \\ ${ }^{4}$ Institute of Biochemistry, Molecular Biology and Biotechnology, University of Colombo, \\ 90 Cumarathunga Munidasa Mawatha, 00300 Colombo 3, Sri Lanka \\ ${ }^{5}$ Department of Biochemistry and Molecular Biology, Faculty of Medicine, University of Colombo, 25 Kynsey Road, \\ 00800 Colombo 8, Sri Lanka \\ ${ }^{6}$ Electrical Engineering Division, Department of Engineering, University of Cambridge, 9 JJ Thomson Avenue, \\ Cambridge CB3 OFA, UK
}

Correspondence should be addressed to Nuwanthi P. Katuwavila; nanukanp@yahoo.com

Received 12 May 2016; Revised 22 July 2016; Accepted 28 July 2016

Academic Editor: Yasuhiko Hayashi

Copyright (C) 2016 Nuwanthi P. Katuwavila et al. This is an open access article distributed under the Creative Commons Attribution License, which permits unrestricted use, distribution, and reproduction in any medium, provided the original work is properly cited.

A chitosan-alginate nanoparticle system encapsulating doxorubicin (DOX) was prepared by a novel ionic gelation method using alginate as the crosslinker. These nanoparticles were around $100 \mathrm{~nm}$ in size and more stable with higher positive zeta potential and had higher \% encapsulation efficiency (95\%) than DOX loaded chitosan nanoparticles (DOX Csn NP) crosslinked with sodium tripolyphosphate (STPP). FTIR spectroscopy and thermogravimetric analysis revealed successful loading of DOX. In vitro drug release showed an initial release phase followed by slow release phase with higher cumulative release obtained with DOX loaded chitosan-alginate nanoparticles (DOX Csn-Alg NP). The in vitro cytotoxicity of DOX released from the two nanoparticle systems showed a notable difference on comparison with that of free DOX on the MCF-7 cell line. The SRB assay, AO/EB staining, and fluorescence uptake study indicated that free DOX only showed dose dependent cytotoxicity, whereas both dose and time dependency were exhibited by the two sets of NPs. While both systems show sustained release of DOX, from the cell viability plots, DOX Csn-Alg NPs showed their superiority over DOX Csn NPs. The results obtained are useful for developing DOX Csn-Alg NPs as a sustained release carrier system for DOX.

\section{Introduction}

Doxorubicin (DOX), with the trade name Adriamycin also known as hydroxydaunorubicin and hydroxydaunomycin, is a drug used in cancer chemotherapy and derived by chemical semisynthesis from a bacterial species [1]. DOX, an anthracycline antibiotic, has been used for treatment of leukemias, Hodgkin's lymphoma, bladder, breast, stomach, lung, ovarian and thyroid cancers, soft tissue sarcoma, and multiple myeloma [2]. The clinical usage of DOX is associated with increased risk of cardiotoxicity which in turn leads to congestive heart failure [3]. In order to increase the anticancer efficacy by prolonging the circulation time in blood stream and reduce its toxicity, different carrier systems for DOX have been developed such as liposomes [4] and polymeric nanoparticles [5]. The primary goal of using nanotechnology in medicine is to develop new safety delivery systems having reduced toxicity and biocompatibility while maintaining therapeutic effects [6].

At present, it has become customary to use naturally occurring polymers as source materials for drug delivery [7]. Nanoparticles of polymers not only protect the bioactive 
material but also facilitate the control release of the material [8]. Due to some inherent problems such as low encapsulation efficiency, and rapid leakage of entrapped drugs in liposomes, usage of polymeric nanoparticles has gained more attention over liposomes [9]. Polysaccharides of natural origin which offer a wide diversity in their structure and properties due to their wide range of molecular weight and chemical composition [10] are extensively being researched in the drug delivery field. Further, the unique properties of polysaccharides such as nontoxicity, full biodegradability, biocompatibility, high stability, hydrophilicity, and also the high abundance in nature and low cost in processing will increase their usage in synthesizing drug delivery systems [11].

Among these naturally occurring polysaccharides, the two chosen, chitosan and alginate, are polyelectrolyte polymers of opposite charges [12]. They are very promising and have been extensively exploited in the drug delivery field for controlled drug release $[13,14]$. Chitosan, a cationic, linear nitrogenous polysaccharide composed of glucosamine and $\mathrm{N}$ -acetyl-glucosamine linked by $(1 \rightarrow 4) \beta$-glycosidic bonds, is the second most abundant polymer in nature after cellulose [15]. It is a hydrophilic polymer obtained from deacetylation of aminoacetyl groups of chitin which is the main component of the shells of crustaceans, the cell walls of the fungi, and the cuticle of insects [16]. In addition to the main properties of polysaccharides such as biocompatibility and biodegradability [17], the bioadhesiveness of chitosan [18] which facilitates the ionic interaction of positively charged amino groups of chitosan with negatively charged mucous layer [19] is accountable for its usage as a promising matrix in pharmaceutical industry. Alginate is a hydrophilic anionic copolymer which is composed of alternating blocks of (1-4) linked $\beta$ $\mathrm{D}$-mannuronic acid ( $\mathrm{M}$ units) and $\alpha$-L-guluronic acid (G units) obtained from natural sources such as cell walls and intercellular spaces of marine brown algae and bacteria [19]. The wide pharmaceutical applicability of alginate depends on its ability to form hydrogels by chelating with divalent cations [20]. The easy gelling property of both chitosan and alginate can be used to prepare polyelectrolyte complexes of polycations and polyanions [21]. Chitosan-alginate polyionic complex is formed via the ionic interactions between the amine group of chitosan and the carboxylic group of alginate through ionic gelation [22]. Since these interactions reduce the porosity of the complex it protects the encapsulant and slows the release effectively than either chitosan or alginate alone [23]. The high solubility of chitosan in low $\mathrm{pH}$ is reduced by the poor solubility of alginate network at low $\mathrm{pH}$, while alginate is stabilized at high $\mathrm{pH}$ by chitosan which is less soluble at high $\mathrm{pH}$ [11].

In the present study, a novel carrier system for DOX was developed with chitosan and alginate. Several researchers have investigated the delivery properties of chitosan-alginate NPs and nanocomposites for a variety of drugs. Though DOX in chitosan NPs have been investigated in various aspects, there is no reported literature on chitosan-alginate NP which was directly used as a delivery vehicle for DOX. There are several reports where chitosan and alginate have been utilized to prepare carriers to deliver DOX. In one method, CMC-predoped porous $\mathrm{CaCO}_{3}$ microparticles were assembled in layered fashion by coating alternatively with chitosan and alginate [24]. A second group had synthesized chitosan-alginate capsules consisting of BSA in a gel capsule for DOX delivery [25]. There is another study in which mesoporous silica nanoparticles were coated with chitosan and alginate to deliver DOX [26]. In the method we report, we have only used the two biopolymers chitosan and alginate to form nanoparticles of a very small size range using a different method following the ionic gelation technique. A comparison with DOX Csn NP revealed an increase in efficiency and potency of Csn-Alg NPs over that of Csn NPs. The cytotoxicity effect of these DOX encapsulated NPs versus free DOX was investigated with the human breast cancer cell line, MCF-7.

\section{Materials and Methods}

2.1. Materials. Chitosan (medium molecular weight, deacetylation degree $\sim 75 \%$ ), low viscosity sodium alginate, sodium tripolyphosphate (STPP), and doxorubicin hydrochloride $(98.0 \%)$ were purchased from Sigma-Aldrich Chemical Company (St. Louis, MO, USA). All other reagents were of analytical grade and used directly.

MCF-7 cells were purchased from ATCC, USA. Powdered Dulbecco's Modified Eagle Medium was purchased from Invitrogen Life Technologies (Carlsbad, CA, USA). Fetal bovine serum (FBS), streptomycin/penicillin, dimethyl sulfoxide (DMSO), agarose, and trypsin/EDTA were purchased from Sigma Aldrich Chemical Company (St. Louis, MO, USA).

\subsection{Preparation of DOX Loaded Chitosan-Alginate Nanopar-} ticles. DOX Csn-Alg NPs were prepared by a novel method. The chitosan flakes were dissolved in $40 \mathrm{~mL}$ of acetic acid $(0.3 \% \mathrm{v} / \mathrm{v})$ and the resulting solution $(2 \mathrm{mg} / \mathrm{mL})$ was adjusted to $\mathrm{pH} 4.8$. The alginate solution $(1 \mathrm{mg} / \mathrm{mL})$ was prepared by dissolving in distilled water at room temperature overnight and adjusting the $\mathrm{pH}$ to 5.2. The drug DOX $(30 \mu \mathrm{g} / \mathrm{mL})$ was stirred with alginate solution for $24 \mathrm{~h}$ to form the drugalginate complex. The chitosan solution was stirred with Tween $80(0.310 \mathrm{~g})$ at $60^{\circ} \mathrm{C}$ for $2 \mathrm{~h}$ to obtain a homogeneous mixture and this solution was gradually dropped to the alginate-DOX complex over $1 \mathrm{~h}$ while stirring at a high speed. The stirring was continued for another half an hour and then refrigerated overnight. The nanoparticle suspension was centrifuged at $9000 \mathrm{rpm}$ for $45 \mathrm{~min}$ to obtain the nanoparticle pellet. Five formulations were prepared by varying chitosan content to obtain different weight ratios of chitosan to alginate of $0.5: 1,0.6: 1,0.8: 1,1: 1$, and $2: 1$.

For the comparison, DOX Csn NP was prepared according to a method modified in literature [27] employing a two-step method, that is, oil in water emulsion followed by crosslinking.

2.3. Characterisation of DOX Loaded Nanoparticles. The average particle size and size polydispersity of the NPs dispersed in water were determined by dynamic light scattering 
technique at $25^{\circ} \mathrm{C}$ using a particle size analyzer (Zetasizer Nano ZS, Malvern Instruments, UK) at a fixed scattering angle of $90^{\circ}$. The zeta potential of nanoparticles was measured using the zeta potential analyzer (Zetasizer Nano ZS, Malvern Instruments, UK). All measurements were performed in triplicate. Fourier Transform Infrared (FTIR) spectra of chitosan, sodium alginate, unloaded nanoparticles, and DOX loaded NPs were obtained with a Bruker Vertex 80 IR spectrometer (Germany) at a resolution of $4 \mathrm{~cm}^{-1}$ from 4000 to $400 \mathrm{~cm}^{-1}$. Thermal decomposition of chitosan, sodium alginate, unloaded nanoparticles, and DOX loaded nanoparticles were analyzed using a SDT Q600 thermogravimetric analyzer (TA Instruments, USA) from $25^{\circ} \mathrm{C}$ to $800^{\circ} \mathrm{C}$ using a ramp rate of $10^{\circ} \mathrm{C} / \mathrm{min}$ in air. Nanoparticle morphology was examined by electron microscopy. For scanning electron microscopy, a drop of the particle suspension was placed on a cleaned stub and dried overnight and imaging was done followed by gold sputtering to the specimen by the scanning electron microscope (SEM, SU6600, Hitachi, Japan) operating at 5 and $10 \mathrm{kV}$. Further the morphology was recorded by highresolution transmission electron microscopy (HR-TEM, JEM 2100, JEOL, Japan) operated at accelerating voltage $200 \mathrm{kV}$. The samples prior to their analysis were placed on carbon coated copper grids and dried under ambient conditions.

2.4. Determination of \% Encapsulation Efficiency. The amount of incorporated DOX in the NPs was determined by measuring the fluorescent absorbance of DOX remaining in the supernatant (free DOX) after centrifugation of the nanoparticle mixture using a fluorometer (Fluorolog, Horiba, Japan) with a slit width of $5 \mathrm{~nm}$ and excitation and emission wavelengths at $468 \mathrm{~nm}$ and $550 \mathrm{~nm}$, respectively. A calibration curve was performed with a series of concentrations to obtain the unknown DOX concentrations. The percent encapsulation efficiency (\% EE) was calculated as follows:

$$
\% \mathrm{EE}=\frac{\text { Total DOX }- \text { Free DOX }}{\text { Total DOX }} \times 100 .
$$

2.5. Evaluation of In Vitro DOX Release. The release characteristics of DOX from polymeric NPs were studied in distilled water and phosphate buffered saline solution (PBS) $(\mathrm{pH} \sim 7.4)$. The centrifuged nanoparticle pellet was dispersed in $5 \mathrm{~mL}$ of $\mathrm{PBS}$ at $\mathrm{pH} 7.4$ and trapped inside a dialysis membrane (cutoff molecular weight $3.5 \mathrm{kDa}$ ) and immersed in $25 \mathrm{~mL}$ PBS. The temperature of the setup was maintained at $37^{\circ} \mathrm{C}$ in the dark with mild agitation. Aliquots $(3 \mathrm{~mL})$ were withdrawn at predetermined time intervals and the fluorescence emission spectrum was recorded as described previously. The release medium was refreshed with $3 \mathrm{~mL}$ of medium after each withdrawal. The release experiments were repeated three times and average data were reported. Using the calibration curve the unknown concentrations were calculated; hence the cumulative release of DOX was determined.

2.6. Cell Culture and In Vitro Cytotoxicity Studies. The cytotoxicity activities of DOX Csn and DOX Csn-Alg NPs against MCF-7 cells were compared to free DOX. MCF7 cells were grown in monolayer cultures in Dulbecco's Modified Eagle Medium (DMEM) supplemented with 10\% fetal bovine serum and $50 \mathrm{IU} / \mathrm{mL}$ penicillin and $50 \mu \mathrm{g} / \mathrm{mL}$ streptomycin. Cells were maintained at $37^{\circ} \mathrm{C}$ in $95 \%$ air $/ 5 \%$ $\mathrm{CO}_{2}$ atmosphere, with $95 \%$ humidity. Culture medium was changed every 2-3 days. Next, the cells in exponential growth were removed by trypsinization and seeded at $5 \times 10^{3}$ cells per well into a 96-well plate and incubated for $24 \mathrm{~h}$ for attachment. After the incubation period, cells were exposed to both DOX loaded NPs (DOX concentration of $0.01,0.02$, $0.05,1.0,4.0$, and $8.0 \mu \mathrm{g} / \mathrm{mL}$ ) and to free DOX with similar concentrations for 24,48 , and $72 \mathrm{~h}$ after incubation.

Cell viability was assessed by sulforhodamine B (SRB) assay [28] and the results were expressed as a percentage of control values. All the assays were performed in triplicate in three different experiments.

2.7. Morphological Observation and Apoptosis Induced by DOX Loaded Nanoparticles. Apoptosis was identified morphologically by acridine orange/ethidium bromide (AO/EB) staining. MCF-7 cells were seeded separately in 24-well plates at $5 \times 10^{4}$ cells $\mathrm{mL}^{-1}$ on cell culture treated coverslips for $24 \mathrm{~h}$ in a humidified atmosphere at $37^{\circ} \mathrm{C}$ in $5 \% \mathrm{CO}_{2}$ to allow cell adherence. Cells were then treated with both types of DOX loaded NPs (DOX concentration of 0.01, 1.00, and $8.00 \mu \mathrm{g} / \mathrm{mL})$ and free $\operatorname{DOX}(0.01,1.00$, and $8.00 \mu \mathrm{g} / \mathrm{mL})$. Treated cells were incubated for 24,48 , and $72 \mathrm{~h}$ and, at the end of each incubation process, cells on the coverslips were observed under an inverted phase contrast microscope (Olympus CKX41SF, Japan) for morphological changes. Cells were then fixed with $4 \%$ formaldehyde in PBS at room temperature for $15 \mathrm{~min}$. Cocktail of acridine orange-ethidium bromide (AO/EB) dyes in PBS, containing $50 \mathrm{mg} \mathrm{mL}^{-1}$ each, was added to the fixed cells on coverslips and incubated for $10 \mathrm{~min}$ at room temperature in the dark. Cells were then visualized under a fluorescence microscope (Olympus, BX51TRF, Japan). Both assays were performed in triplicate in three different experiments.

2.8. Cellular Uptake of Nanoparticles. MCF-7 cells were seeded separately in 24 -well plates at $5 \times 10^{4}$ cells $\mathrm{mL}^{-1}$ on coverslips for $24 \mathrm{~h}$ in a humidified atmosphere at $37^{\circ} \mathrm{C}$ in $5 \%$ $\mathrm{CO}_{2}$ to allow cell adherence. Cells were then treated with both DOX loaded NP systems (DOX concentration of 0.01, 1.00, and $8.00 \mu \mathrm{g} / \mathrm{mL})$ and free DOX $(0.01,1.00$, and $8.00 \mu \mathrm{g} / \mathrm{mL})$. Treated cells were incubated for $30 \mathrm{~min}$ and 2 and $24 \mathrm{~h}$ and at the end of each incubation process cells were rinsed twice with PBS and then fixed with $4 \%$ formaldehyde in PBS at room temperature for 15 mins. The cells on the coverslips were observed under the fluorescence microscope (Olympus BX51TRF, Japan).

\section{Results and Discussion}

The main effort of this work was to develop a nanodelivery system for the drug DOX using natural, biodegradable, biocompatible polysaccharides specially chitosan and alginate. 
TABLE 1: The size, zeta potential, and \% EE of DOX Csn and DOX Csn-Alg NPs. All analyses were performed using the DOX loaded NPs prepared by using optimized formulations (chitosan: STTP $2: 1$ and chitosan : alginate $2: 1$ ).

\begin{tabular}{lcccc}
\hline System & Average size/nm & Zeta potential/mV & \% Encapsulation efficiency & Polydispersity index \\
\hline DOX Csn NPs & $100 \pm 27$ & $26 \pm 3$ & $65 \pm 4$ & $0.391 \pm 0.048$ \\
DOX Csn-Alg NPs & $100 \pm 35$ & $35 \pm 4$ & $95 \pm 4$ & $0.402 \pm 0.071$ \\
Csn NPs & $100 \pm 25$ & $30 \pm 5$ & - & $0.352 \pm 0.046$ \\
Csn-Alg NPs & $100 \pm 28$ & $36 \pm 3$ & - & $0.414 \pm 0.065$ \\
\hline
\end{tabular}

The feasibility of using Csn-Alg NPs as a delivery system for DOX was evaluated with comparison to DOX Csn NPs.

3.1. Formation of DOX Loaded Polymeric Nanoparticles. The chitosan NPs are easily formed by the gelation with STPP via electrostatic interactions. At a $\mathrm{pH}$ around 4.8 the protonated amino groups of chitosan interact electrostatically with negatively charged phosphate groups of STPP [29]. The uncertainty of this encapsulation was the complexation of DOX into the positively charged chitosan since DOX also exhibits a positive charge. However, 65\% of DOX was encapsulated into Csn NPs in this experiment, overcoming the charge repulsion. Since Csn NPs have shown positive results for the DOX delivery, an effort was taken to improve the DOX delivery with the use of alginate polymer as well. The polyelectrolyte complex Csn-Alg NPs was prepared using a novel method. Initially, the chitosan droplet formation occurred with the stirring with Tween 80 , followed by the droplet solidification by ionically crosslinking of the alginate solution. In aqueous solutions, at $\mathrm{pH}$ around 4 to 5.5 the amine groups on chitosan became easily positively charged. Alginate, on the other hand, dissolved in a neutral $\mathrm{pH}$ solution where the carboxylate groups were negatively charged. In aqueous solutions with $\mathrm{pH}$ around 5.2 chitosan amino groups interacted with alginate carboxylate groups to form the hydrogel. Since alginate is a negatively charged polymer the positively charged DOX was complexed initially with alginate to obtain a higher loading of the drug to the nanoparticle. The formulation, (chitosan: sodium alginate, 2:1), which gave an opalescent suspension with a positive zeta potential, was selected as the optimum ratio for the DOX encapsulation. This formulation resulted in $95 \%$ encapsulation efficiency of DOX.

3.2. Shape, Size, and Surface Charge of Doxorubicin Loaded Nanoparticles. The morphology of the polymeric NPs was observed by scanning electron microscope and Transmission Electron Microscope and both NPs were below $20 \mathrm{~nm}$ and spherical in shape (Figures 1 and 2). Further, the TEM images clearly showed the well separated, well dispersed particles. After loading of DOX, there was no noticeable change either in the shape or in the size in SEM and TEM images of both NPs. In a study on carvacrol-loaded chitosan NPs, Keawchaoon and Yoksan have reported TEM images showing 40-80 nm sized NPs [27]. In a previous study done by Liu et al., the particle sizes of Csn-Alg NPs obtained from the transmission electron microscopy (TEM) were 20-50 nm [11].
A study by Motwani et al. had shown the particle sizes of Csn-Alg NPs obtained from TEM to be around $63 \mathrm{~nm}$ [22]. Thus, using the above-mentioned method we were able to obtain much smaller Csn-Alg NPs. The sizes obtained from DLS technique were larger $(\sim 100 \mathrm{~nm}$, Table 1$)$ than the sizes obtained from SEM and TEM since DLS measures the hydrodynamic diameter. Also the larger particle size might be due to the swellability of polymeric hydrogels in the solution [30] whereas electron microscopy shows the images of dried particles.

The zeta potentials of both DOX Csn and DOX CsnAlg NPs show large positive values reflecting the stability of the colloidal suspensions (Table 1). Having positively charged surfaces is an added advantage when using NPs in drug delivery since they are able to transfer easily through negative channels in the cell membrane. An increased zeta potential value with DOX Csn-Alg NPs, when compared to the DOX Csn NPs, was caused by the higher amount of positively charged DOX encapsulation with Csn-Alg NPs than that with Csn NPs. Neutralization of the charge on chitosan by the strong negative charge of STTP versus alginate at the working $\mathrm{pH}$ may have also contributed to the differences in zeta potential.

3.3. Characterisation of DOX Loaded Nanoparticles. The successful loading of DOX into the polymeric NPs was confirmed by FTIR and TGA techniques. Chemical structures of both reactants and products were analyzed by FTIR spectroscopy. In the spectra of pure chitosan (Figure 3(b)), characteristic peaks at $3449 \mathrm{~cm}^{-1}$ for $-\mathrm{NH}_{2}$ and $-\mathrm{OH}$ stretching, $1659 \mathrm{~cm}^{-1}$ for $-\mathrm{CO}$ stretching, and $1596 \mathrm{~cm}^{-1}$ for $-\mathrm{NH}_{2}$ bending vibrations were observed. Crosslinking of the amine group of chitosan with phosphate group of STPP depicts a clear shift in the peak at $3565 \mathrm{~cm}^{-1}$ for $-\mathrm{NH}_{2}$ and $-\mathrm{OH}$ stretching as shown in the spectrum of Csn NPs (Figure 3(c)). On loading DOX into Csn NPs, the FTIR spectra (Figure 3(e)) show peak shifts resulting from hydrogen bonding in the above modes, that is, $3578 \mathrm{~cm}^{-1}$ for $-\mathrm{NH}_{2}$ and $-\mathrm{OH}$ stretching, $1685 \mathrm{~cm}^{-1}$ for $-\mathrm{CO}$ stretching, and $1578 \mathrm{~cm}^{-1}$ for $-\mathrm{NH}_{2}$ bending vibrations reflecting the successful loading of DOX into the Csn NPs.

In the spectra of pure chitosan the major vibrations of $-\mathrm{NH}_{2}$ and $-\mathrm{OH}$ stretching peak appearing at $3449 \mathrm{~cm}^{-1}$ had shifted to $3565 \mathrm{~cm}^{-1}$ in the spectra of Csn-Alg NPs and the $-\mathrm{CO}$ stretching vibration at $1659 \mathrm{~cm}^{-1}$ in pure chitosan had also shifted to $1651 \mathrm{~cm}^{-1}$ after interaction of the amine group of chitosan with the carboxylic group of alginate 


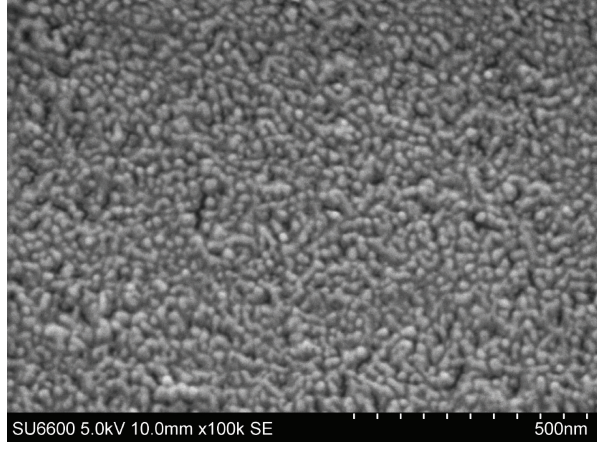

(a)

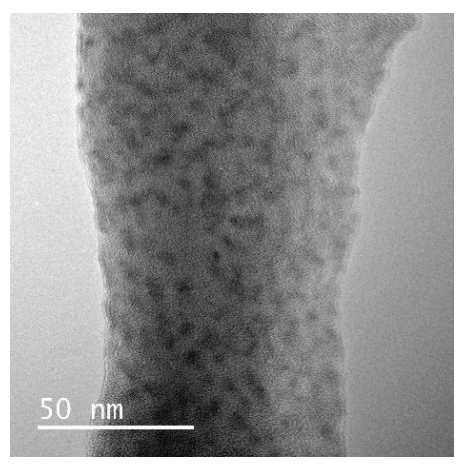

(b)

Figure 1: (a) Scanning electron microscopy image of Csn NP. (b) Transmission electron microscopy image of Csn NP.

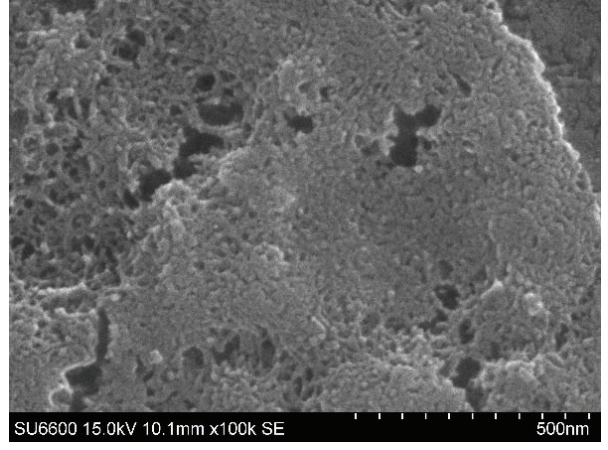

(a)

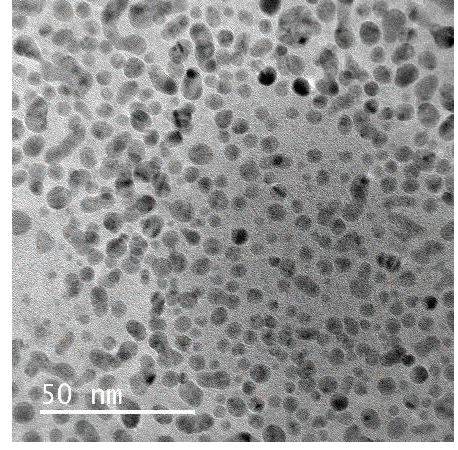

(b)

Figure 2: (a) Scanning electron microscopy image of Csn-Alg NP. (b) Transmission electron microscopy image of Csn-Alg NP.

(Figure 3(d)). Further, the $-\mathrm{NH}_{2}$ bending peak at $1596 \mathrm{~cm}^{-1}$ disappeared due to binding with alginate to form the NP. After loading DOX into the Csn-Alg NPs, the $-\mathrm{NH}_{2}$ and $-\mathrm{OH}$ stretching peak at $3565 \mathrm{~cm}^{-1}$ and the $-\mathrm{CO}$ stretching vibration at $1660 \mathrm{~cm}^{-1}$ shifted to $3356 \mathrm{~cm}^{-1}$ and to $1637 \mathrm{~cm}^{-1}$, respectively. Further, the appearance of a small peak at $1542 \mathrm{~cm}^{-1}$ for $-\mathrm{NH}_{2}$ bending, after loading the DOX, may reflect the physical entrapment of DOX into the Csn-Alg NPs (Figure 3(f)).

\subsection{Thermal Behaviour of DOX Loaded Polymeric Nanopar-} ticles. Nanoparticles with and without DOX were analyzed by thermogravimetry to further evaluate the thermal stability and successful loading of DOX. In the thermogram of chitosan NPs, a major one-step mass loss about $40 \%$ of total weight was observed at a peaking temperature (inflection point temperature) of $216^{\circ} \mathrm{C}$ (Figure 4). Heating up to $800^{\circ} \mathrm{C}$ retained $35 \%$ of the weight of the nanoparticles from the residual sodium ions of the STPP crosslinked Csn NPs. Similarly, in DOX loaded chitosan nanoparticles a 50\% weight loss from $100-400^{\circ} \mathrm{C}$ was observed in two steps due to the combined decomposition of chitosan and DOX but the peaking temperature of $30 \%$ weight loss had shifted to $225^{\circ} \mathrm{C}$ proving the loading of the DOX into chitosan nanoparticles (Figure 4). A new peak with $12 \%$ weight loss in the temperature range $600-700^{\circ} \mathrm{C}$ was observed with the DOX Csn NPs showing entrapment of DOX into the polymer matrix.

The decomposition of unloaded Csn-Alg NPs had two major and two minor weight losses (Figure 4). The thermogram of DOX loaded NPs showed the same pattern of weight loss as the unloaded particles. However, when the temperature reached $350^{\circ} \mathrm{C}, 60 \%$ of weight loss was observed with DOX loaded nanoparticles due to the decomposition of DOX complexed nanoparticles, whereas it was only $50 \%$ with unloaded nanoparticles. Further, the inflection point temperature of the second major step of weight loss of unloaded composite in the range of $600-750^{\circ} \mathrm{C}$ peaked at $703^{\circ} \mathrm{C}$, whereas in the DOX loaded nanocomposite it peaked at $750^{\circ} \mathrm{C}$ reflecting the strong physical entrapment of DOX into the nanoparticles.

3.5. In Vitro Release Kinetics of DOX from Polymeric Nanoparticles. The in vitro release profiles of both optimized formulations in phosphate buffered saline (PBS) solution (pH-7.4) are shown in Figure 5. An initial burst release of DOX from both NPs in PBS solution can be observed up to $24 \mathrm{~h}$. This accounts for about $40 \%$ of DOX from the total encapsulated amount. Then, it followed a more gradual and 


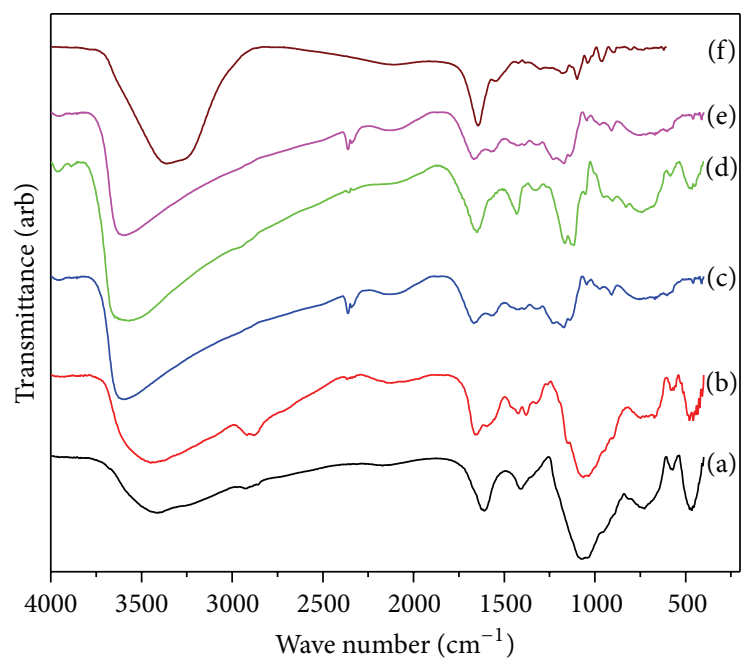

FIGURE 3: FTIR spectra of (a) pure alginate, (b) pure chitosan, (c) Csn NP prepared using $2: 1$ weight ratio of chitosan to STPP, (d) CsnAlg NP prepared using 2:1 weight ratio of chitosan to alginate (e) DOX Csn NP, and (f) DOX Csn-Alg NP.

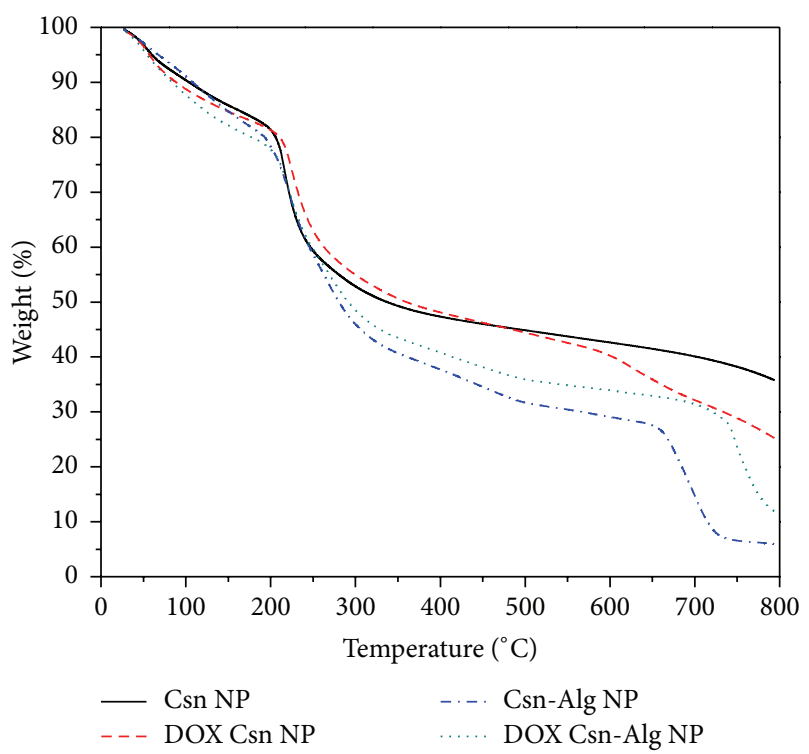

FIgURE 4: TGA thermograms of Csn NP, DOX Csn NP prepared using 2:1 weight ratio of chitosan to STPP and Csn-Alg NP, and DOX Csn-Alg NP prepared using 2:1 weight ratio of chitosan to alginate.

sustained release phase for the next $72 \mathrm{~h}$. Both profiles had followed the same pattern of release but the amounts were different. The total amount of drug released from Csn-Alg NPs was around $60 \%$. In contrast, the total amount of drug released from Csn NPs was around 45\%. These results clearly indicated that the release of DOX was retarded significantly due to the encapsulation in nanoparticles.

3.6. In Vitro Cytotoxicity of DOX Loaded Polymeric Nanoparticle. Dose response curves were plotted for 3 formulations,

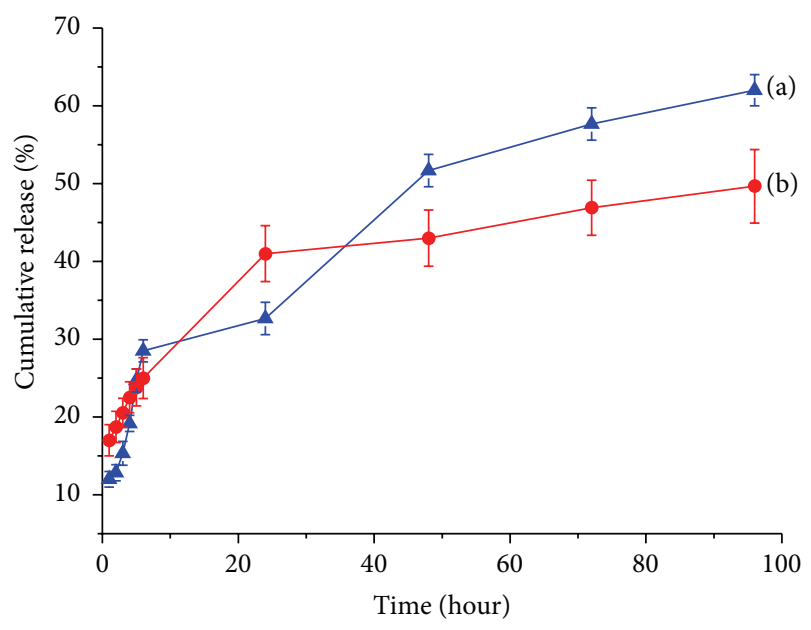

FIgure 5: Cumulative release of DOX from (a) DOX Csn-Alg and (b) DOX Csn NP at preselected time intervals in pH 7.4 PBS. Results were reported as mean $\pm \mathrm{SD}, n=3$.

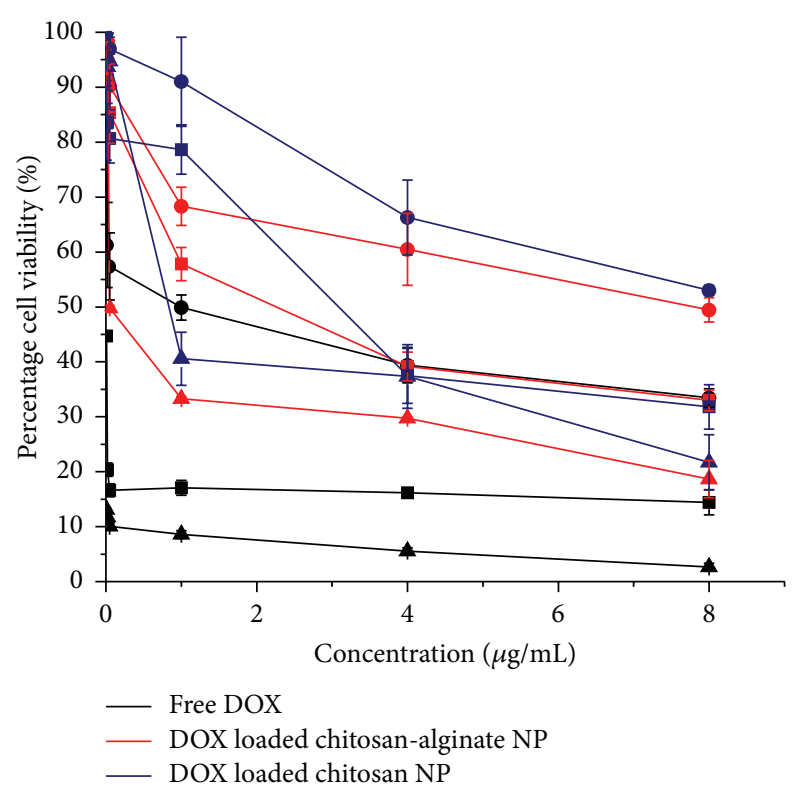

FIgure 6: Cytotoxicity of free DOX, DOX Csn-Alg NP, and DOX Csn NP against MCF-7 cells after $24 \quad 48$ and $\boldsymbol{\Delta} 72$ hours of treatments. Cell survival was assessed via SRB assay. Results were reported as mean $\pm \mathrm{SD}, n=4$.

free DOX, DOX Csn-Alg NPs, and DOX Csn NPs (Figure 6). Each experimental curve represents the average of a series of 3 experiments. With free DOX, the inhibition of MCF-7 cell proliferation completely depends on the dose of the drug used. After 48 and $72 \mathrm{~h}, 50 \%$ cell viability could not be observed even at the lowest concentration $(0.01 \mu \mathrm{g} / \mathrm{mL})$ of free DOX, proving the reduced effect of free DOX after $24 \mathrm{~h}$; that is, the free DOX has dose dependent cytotoxicity; thus it might damage the normal cells during therapy, whereas the current work demonstrated the dose-dependence as well as 


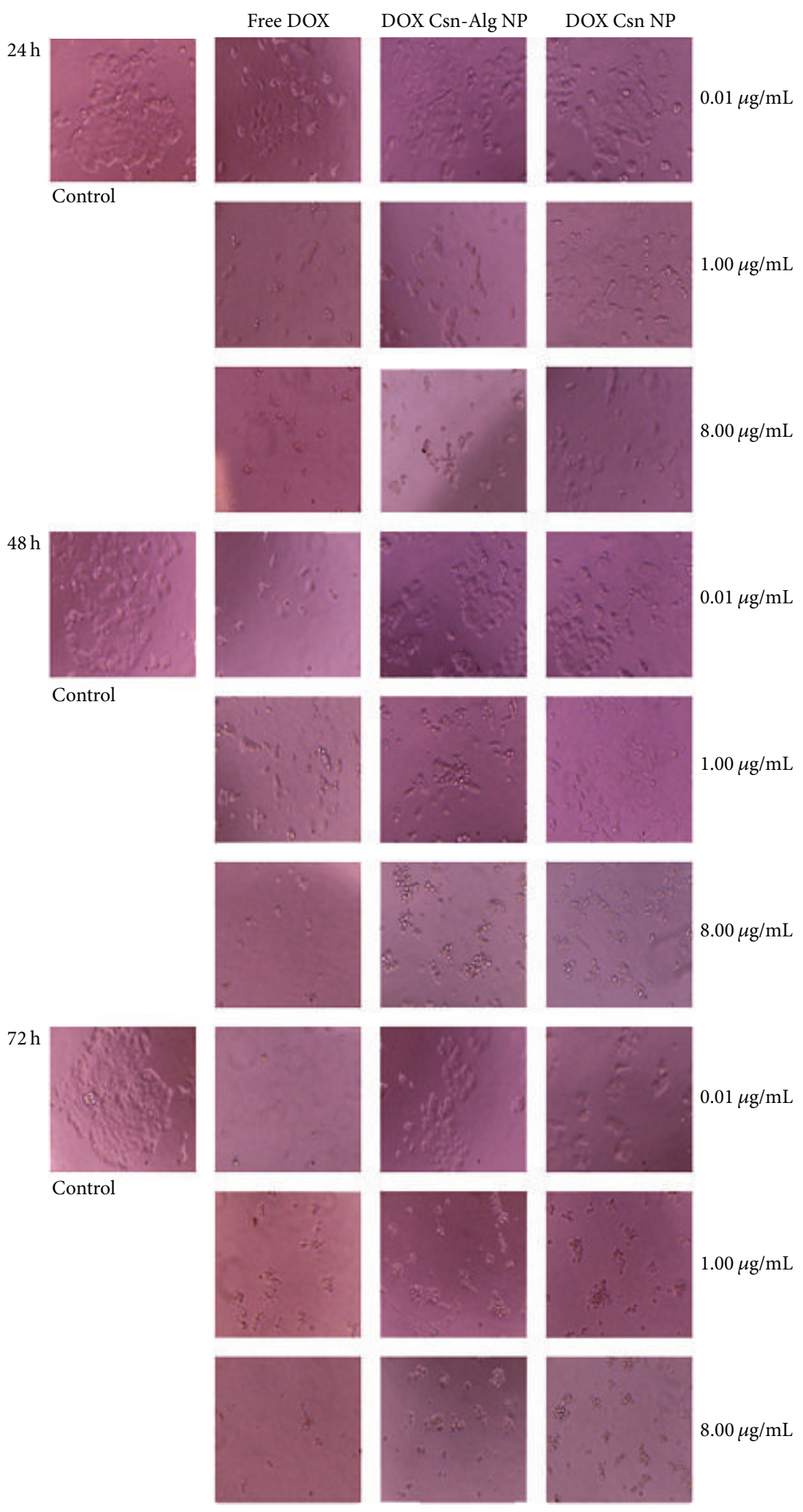

FIGURE 7: Morphological changes of apoptosis in MCF-7 cells observed under inverted light microscopy exposed to different concentrations of free DOX, DOX Csn-Alg NP, and DOX Csn NP after $24 \mathrm{~h}, 48 \mathrm{~h}$, and $72 \mathrm{~h}$ after incubation. 


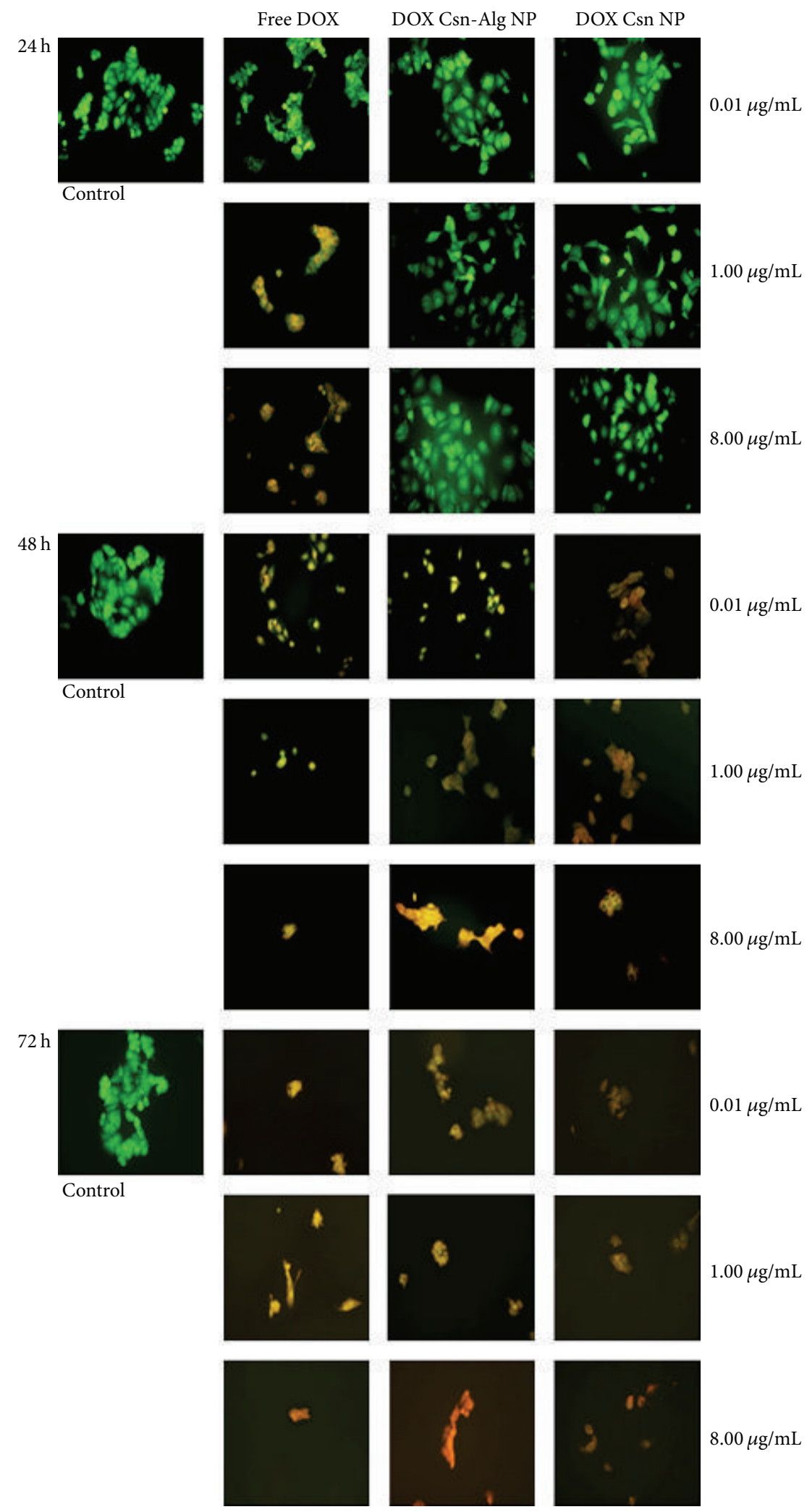

FIGURE 8: Fluorescence microscopic observations of MCF-7 cells treated with different concentrations of free DOX, DOX Csn-Alg NP, and DOX Csn NP stained with AO/EB after $24 \mathrm{~h}, 48 \mathrm{~h}$, and $72 \mathrm{~h}$ after incubation. 


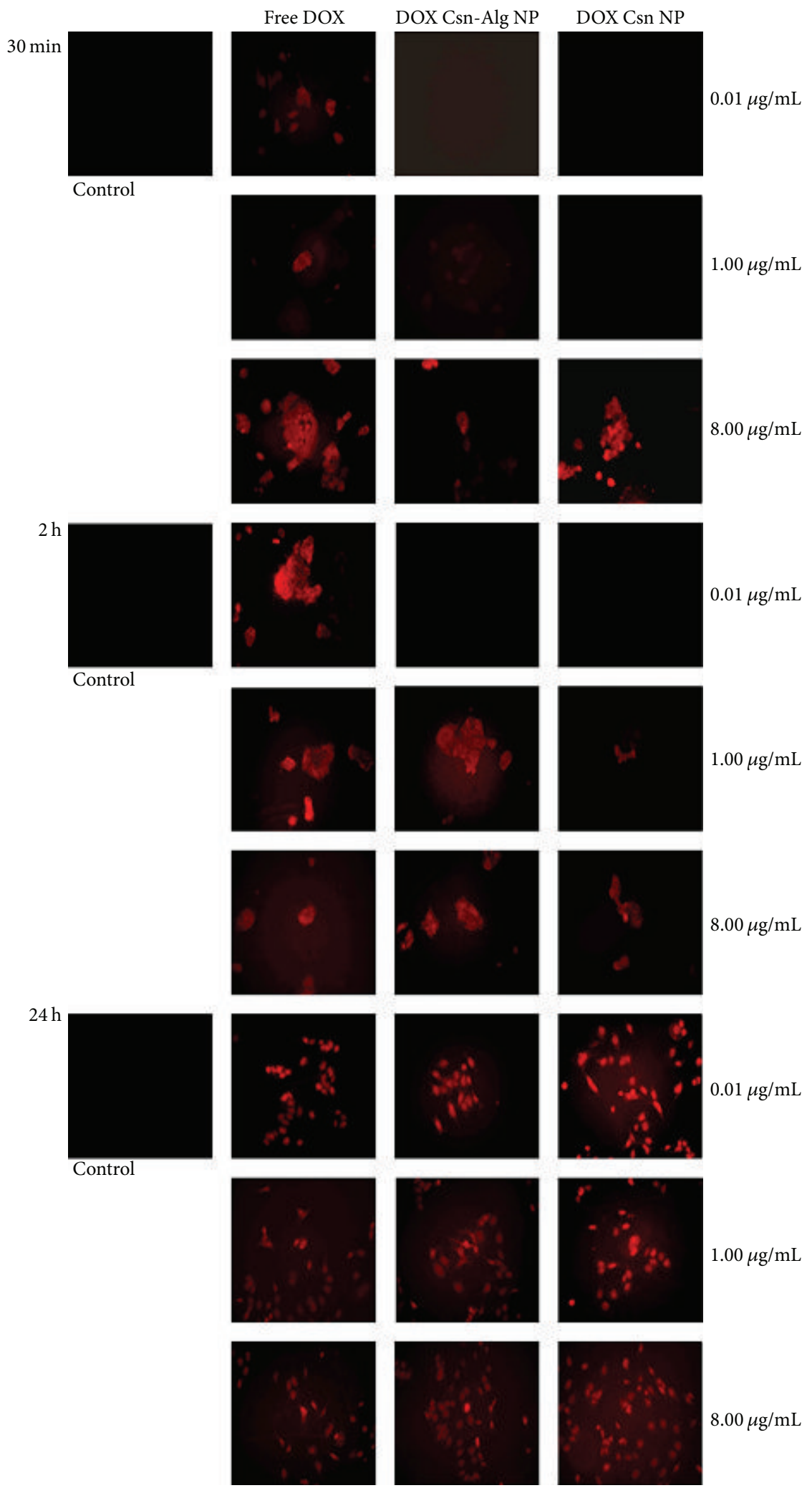

FIGURE 9: Fluorescence microscopic observations of cellular uptake of MCF-7 cells treated with different concentrations of free DOX, DOX Csn-Alg NP, and DOX Csn NP after $30 \mathrm{~min}, 2 \mathrm{~h}$, and $24 \mathrm{~h}$ after incubation. 
the time dependence cytotoxicity of DOX loaded NPs with MCF-7 cell line. None of the concentrations of DOX in both NPs that were used caused $50 \%$ inhibition of cell growth at $24 \mathrm{~h}$ reflecting the slow release from NPs. Hence, during the first $24 \mathrm{~h}$, those NPs are capable of circulating in the blood and accumulating at tumour sites before starting their cytotoxic effect. Thus, DOX encapsulated in NPs achieve its cytotoxicity with time; that is, there is an effect of both time and dose on the inhibition of cell proliferation. Therefore, this result clearly indicated that the release of DOX was retarded significantly by the encapsulation in NPs. When comparing the two delivery systems, the cytotoxicity effect of DOX Csn NPs was consistently lower than that of DOX Csn-Alg NPs (Figure 6). This is in accordance with the results of in vitro release of DOX being higher from Csn-Alg NPs than from Csn NPs after $72 \mathrm{~h}$ (Figure 5). However, both DOX loaded systems exhibited similar behaviour with the MCF-7 cell line (Figure 6). Same concentration of DOX when encapsulated in NPs behaves differently than in the free state; that is, encapsulated DOX releases slowly and gradually increases the concentration in the medium. Hence, it proves that encapsulating DOX in either system has improved the efficacy of DOX over free DOX. A similar study of doxorubicin loaded magnetic NPs grafted to smart copolymers showed that free DOX has dose dependent but not time dependent cytotoxicity but DOX loaded magnetic NPs have time dependent cytotoxicity [31].

Inverted phase contrast microscopy was used for morphological analysis of MCF-7 cells for observation of apoptosis induced by the DOX loaded NPs and free DOX. These characteristic morphological changes of apoptosis with time and dose in MCF-7 cells on treatment after 24, 48, and $72 \mathrm{~h}$ (Figure 7) were clearly visualized. Exposure to free DOX clearly showed a higher cell confluence at low doses while higher concentration of free DOX decreased cell confluence and showed a higher proportion of rounded cells resulting from detached cells from the culture monolayer. Within the first $24 \mathrm{~h}$, most of the cells were damaged by the induced cytotoxicity of free DOX reflecting its dose dependent cytotoxicity. On the other hand, both DOX loaded systems showed disappearance of the high cell confluence with the dose of the drug and with time (Figure 7). This observation is further confirmed by the fluorescence microscopic results of AO/EB stained MCF-7 cells after 24, 48, and $72 \mathrm{~h}$ of incubation, following the treatment with different concentrations of free DOX and with the same concentrations loaded in Csn and Csn-Alg NPs. Nuclei of viable cells were stained uniformly bright green by $\mathrm{AO}$ while the apoptotic cells exhibited yellow to orange coloration depending on the degree of loss of membrane integrity, due to costaining with EB (Figure 8). Early apoptotic cells have greenish yellow nuclei while late apoptotic cells have orange to red nuclei with condensed or fragmented chromatin (Figure 8). These figures clearly displayed the difference in behaviour of DOX when encapsulated in NPs. Free DOX has a dose dependent increase in induction of apoptosis (Figure 8-24 h). Both the dose and time dependency of DOX encapsulated in NPs are evident from the alterations in cell staining in all the three series (Figure 8). In the case of DOX loaded NPs, the drug has to be released slowly from the NPs before it can exert an effect on the cancer cells, but free DOX has an immediate effect on the cells since it has direct contact with the cells initially. The mechanism of action of apoptosis of DOX loaded in NPs was not significantly different from that of the free DOX indicating that the encapsulation of DOX in NPs does not make any changes to its mechanism of action of apoptosis.

3.7. Cellular Uptake of Nanoparticles. DOX is a fluorescence active compound, having a bright red color. The red color indicates the cellular uptake of DOX when compared to the untreated cells or cells with no internalized DOX. Internalization of DOX in cancer cells was evaluated on MCF-7 cells at different time intervals and different concentrations of free and encapsulated DOX. Within $30 \mathrm{~min}$ of incubation, all concentrations of free DOX were localized within the cell nucleus (Figure 9, $30 \mathrm{~min}$ ), whereas the localization of DOX in NPs could be visualized depending on the concentration and on time (Figure 9). The efficient uptake of the NPs could be facilitated by the positive surface charge of the NPs. A similar observation was noted with the in vitro cellular uptake of paclitaxel loaded PEGylated PLGA-based NPs [32]. In general, NPs are nonspecifically internalized into cells via endocytosis or phagocytosis while free DOX diffuses through the cell membrane [33].

Morphology analysis suggests that the process of cell growth inhibition by DOX loaded in NPs occurs over a much longer time than by the free DOX. From these observations it could be inferred that the DOX in NPs sufficiently associates with the cells with time.

\section{Conclusion}

This study was made to assess the usefulness of the CsnAlg NP system as a delivery agent for the anticancer drug DOX. Comparison with DOX Csn NPs indicated that CsnAlg NPs were more efficient at entrapment of DOX than the Csn NPs. This successful loading was confirmed by both FTIR and TGA data. Both nanoparticles were less than $100 \mathrm{~nm}$ in size with positive zeta potentials. The in vitro release profiles observed for these NPs were characterized by a burst initial release followed by a more gradual and sustained release phase. The in vitro cytotoxicity studies demonstrated that DOX loaded NPs have potent antigrowth effect on MCF-7 cells and inhibited its cell growth, in a dose and time dependent manner. Further, the observed cell apoptosis induced by these DOX loaded NPs by phase contrast microscopy and fluorescence microscopy after $\mathrm{AO} / \mathrm{EB}$ staining proved that there is no significant change in the mechanism of action of DOX after encapsulating in NPs and reflect the slow release of DOX from the NPs. According to the cytotoxicity results, DOX Csn-Alg NPs are more cytotoxic than DOX Csn NPs.

\section{Competing Interests}

The authors declare that they have no competing interests. 


\section{Acknowledgments}

This research was financially supported by the HETC QIG Window 3 Grant, University of Peradeniya, Peradeniya, Sri Lanka. The authors also thank Ms. Damayanthi Dahanayake at Sri Lanka Institute of nanotechnology for her valuable support in electron microscopy imaging.

\section{References}

[1] N. Lomovskaya, S. L. Otten, Y. Doi-Katayama et al., "Doxorubicin overproduction in Streptomyces peucetius: cloning and characterization of the $\mathrm{dnrU}$ ketoreductase and $\mathrm{dnrV}$ genes and the doxA cytochrome P-450 hydroxylase gene," Journal of Bacteriology, vol. 181, no. 1, pp. 305-318, 1999.

[2] K. M. Laginha, S. Verwoert, G. J. R. Charrois, and T. M. Allen, "Determination of doxorubicin levels in whole tumor and tumor nuclei in murine breast cancer tumors," Clinical Cancer Research, vol. 11, no. 19, pp. 6944-6949, 2005.

[3] G. Minotti, P. Menna, E. Salvatorelli, G. Cairo, and L. Gianni, "Anthracyclines: molecular advances and pharmacologic developments in antitumor activity and cardiotoxicity," Pharmacological Reviews, vol. 56, no. 2, pp. 185-229, 2004.

[4] M. E. R. O’Brien, N. Wigler, M. Inbar et al., "Reduced cardiotoxicity and comparable efficacy in a phase III trial of pegylated liposomal doxorubicin $\mathrm{HCl}\left(\mathrm{CAELYX}^{\mathrm{TM}} /\right.$ Doxil $^{\circledR}$ ) versus conventional doxorubicin for first-line treatment of metastatic breast cancer," Annals of Oncology, vol. 15, no. 3, pp. 440-449, 2004.

[5] C. Zhang, W. Wang, T. Liu et al., "Doxorubicin-loaded glycyrrhetinic acid-modified alginate nanoparticles for liver tumor chemotherapy," Biomaterials, vol. 33, no. 7, pp. 2187-2196, 2012.

[6] W. H. De Jong and P. J. A. Borm, "Drug delivery and nanoparticles: applications and hazards," International Journal of Nanomedicine, vol. 3, no. 2, pp. 133-149, 2008.

[7] G. Vilar, J. Tulla-Puche, and F. Albericio, "Polymers and drug delivery systems," Current Drug Delivery, vol. 9, no. 4, pp. 367394, 2012.

[8] A. Kidane and P. P. Bhatt, "Recent advances in small molecule drug delivery," Current Opinion in Chemical Biology, vol. 9, no. 4, pp. 347-351, 2005.

[9] K. S. Soppimath, T. M. Aminabhavi, A. R. Kulkarni, and W. E. Rudzinski, "Biodegradable polymeric nanoparticles as drug delivery devices," Journal of Controlled Release, vol. 70, no. 1-2, pp. 1-20, 2001.

[10] T. Coviello, P. Matricardi, C. Marianecci, and F. Alhaique, "Polysaccharide hydrogels for modified release formulations," Journal of Controlled Release, vol. 119, no. 1, pp. 5-24, 2007.

[11] Z. Liu, Y. Jiao, Y. Wang, C. Zhou, and Z. Zhang, "Polysaccharides-based nanoparticles as drug delivery systems," Advanced Drug Delivery Reviews, vol. 60, no. 15, pp. 1650-1662, 2008.

[12] O. Borges, G. Borchard, J. C. Verhoef, A. De Sousa, and H. E. Junginger, "Preparation of coated nanoparticles for a new mucosal vaccine delivery system," International Journal of Pharmaceutics, vol. 299, no. 1-2, pp. 155-166, 2005.

[13] Y. Murata, D. Jinno, D. Liu, T. Isobe, K. Kofuji, and S. Kawashima, "The drug release profile from calcium-induced alginate gel beads coated with an alginate hydrolysate," Molecules, vol. 12, no. 11, pp. 2559-2566, 2007.
[14] P. Li, Y.-N. Dai, J.-P. Zhang, A.-Q. Wang, and Q. Wei, “Chitosanalginate nanoparticles as a novel drug delivery system for nifedipine," International Journal of Biomedical Science, vol. 4, no. 3, pp. 221-228, 2008.

[15] C. K. S. Pillai, W. Paul, and C. P. Sharma, "Chitin and chitosan polymers: chemistry, solubility and fiber formation," Progress in Polymer Science, vol. 34, no. 7, pp. 641-678, 2009.

[16] F. Khoushab and M. Yamabhai, "Chitin research revisited," Marine Drugs, vol. 8, no. 7, pp. 1988-2012, 2010.

[17] T. Kean and M. Thanou, "Biodegradation, biodistribution and toxicity of chitosan," Advanced Drug Delivery Reviews, vol. 62, no. 1, pp. 3-11, 2010.

[18] O. Gåserød, I. G. Jolliffe, F. C. Hampson, P. W. Dettmar, and G. Skjåk-Bræk, "The enhancement of the bioadhesive properties of calcium alginate gel beads by coating with chitosan," International Journal of Pharmaceutics, vol. 175, no. 2, pp. 237-246, 1998.

[19] M. George and T. E. Abraham, "Polyionic hydrocolloids for the intestinal delivery of protein drugs: alginate and chitosan-a review," Journal of Controlled Release, vol. 114, no. 1, pp. 1-14, 2006.

[20] A. Saha, R. Yadav, and N. Rajendran, "Biomaterials from sponges, ascidians and other marine organisms," International Journal of Pharmaceutical Sciences Review and Research, vol. 27, no. 2, article no. 17, pp. 100-109, 2014.

[21] S. De and D. Robinson, "Polymer relationships during preparation of chitosan-alginate and poly-l-lysine-alginate nanospheres," Journal of Controlled Release, vol. 89, no. 1, pp. 101-112, 2003.

[22] S. K. Motwani, S. Chopra, S. Talegaonkar, K. Kohli, F. J. Ahmad, and R. K. Khar, "Chitosan-sodium alginate nanoparticles as submicroscopic reservoirs for ocular delivery: formulation, optimisation and in vitro characterisation," European Journal of Pharmaceutics and Biopharmaceutics, vol. 68, no. 3, pp. 513-525, 2008.

[23] F.-L. Mi, H.-W. Sung, and S.-S. Shyu, "Drug release from chitosan-alginate complex beads reinforced by a naturally occurring cross-linking agent," Carbohydrate Polymers, vol. 48, no. 1, pp. 61-72, 2002.

[24] Q. Zhao, B. Han, Z. Wang, C. Gao, C. Peng, and J. Shen, "Hollow chitosan-alginate multilayer microcapsules as drug delivery vehicle: doxorubicin loading and in vitro and in vivo studies," Nanomedicine: Nanotechnology, Biology, and Medicine, vol. 3, no. 1, pp. 63-74, 2007.

[25] H. Shen, H. Shi, M. Xie et al., "Biodegradable chitosan/alginate BSA-gel-capsules for $\mathrm{pH}$-controlled loading and release of doxorubicin and treatment of pulmonary melanoma," Journal of Materials Chemistry B, vol. 1, no. 32, pp. 3906-3917, 2013.

[26] W. Feng, W. Nie, C. He et al., "Effect of pH-responsive alginate/chitosan multilayers coating on delivery efficiency, cellular uptake and biodistribution of mesoporous silica nanoparticles based nanocarriers," ACS Applied Materials \& Interfaces, vol. 6, no. 11, pp. 8447-8460, 2014.

[27] L. Keawchaoon and R. Yoksan, "Preparation, characterization and in vitro release study of carvacrol-loaded chitosan nanoparticles," Colloids and Surfaces B: Biointerfaces, vol. 84, no. 1, pp. 163-171, 2011.

[28] S. R. Samarakoon, I. Thabrew, P. B. Galhena, D. De Silva, and K. Tennekoon, "A comparison of the cytotoxic potential of standardized aqueous and ethanolic extracts of a polyherbal mixture comprised of Nigella sativa (seeds), Hemidesmus indicus (roots) 
and Smilax glabra (rhizome)," Pharmacognosy Research, vol. 2, no. 6, pp. 335-342, 2010.

[29] K. Nagpal, S. K. Singh, and D. N. Mishra, "Chitosan nanoparticles: a promising system in novel drug delivery," Chemical and Pharmaceutical Bulletin, vol. 58, no. 11, pp. 1423-1430, 2010.

[30] N. Bhattarai, J. Gunn, and M. Zhang, "Chitosan-based hydrogels for controlled, localized drug delivery," Advanced Drug Delivery Reviews, vol. 62, no. 1, pp. 83-99, 2010.

[31] A. Akbarzadeh, M. Samiei, S. W. Joo et al., "Synthesis, characterization and in vitro studies of doxorubicin-loaded magnetic nanoparticles grafted to smart copolymers on A549 lung cancer cell line," Journal of Nanobiotechnology, vol. 10, article 46, 2012.

[32] F. Danhier, N. Lecouturier, B. Vroman et al., "Paclitaxel-loaded PEGylated PLGA-based nanoparticles: in vitro and in vivo evaluation," Journal of Controlled Release, vol. 133, no. 1, pp. 11$17,2009$.

[33] A. des Rieux, V. Fievez, M. Garinot, Y.-J. Schneider, and V. Préat, "Nanoparticles as potential oral delivery systems of proteins and vaccines: a mechanistic approach," Journal of Controlled Release, vol. 116, no. 1, pp. 1-27, 2006. 

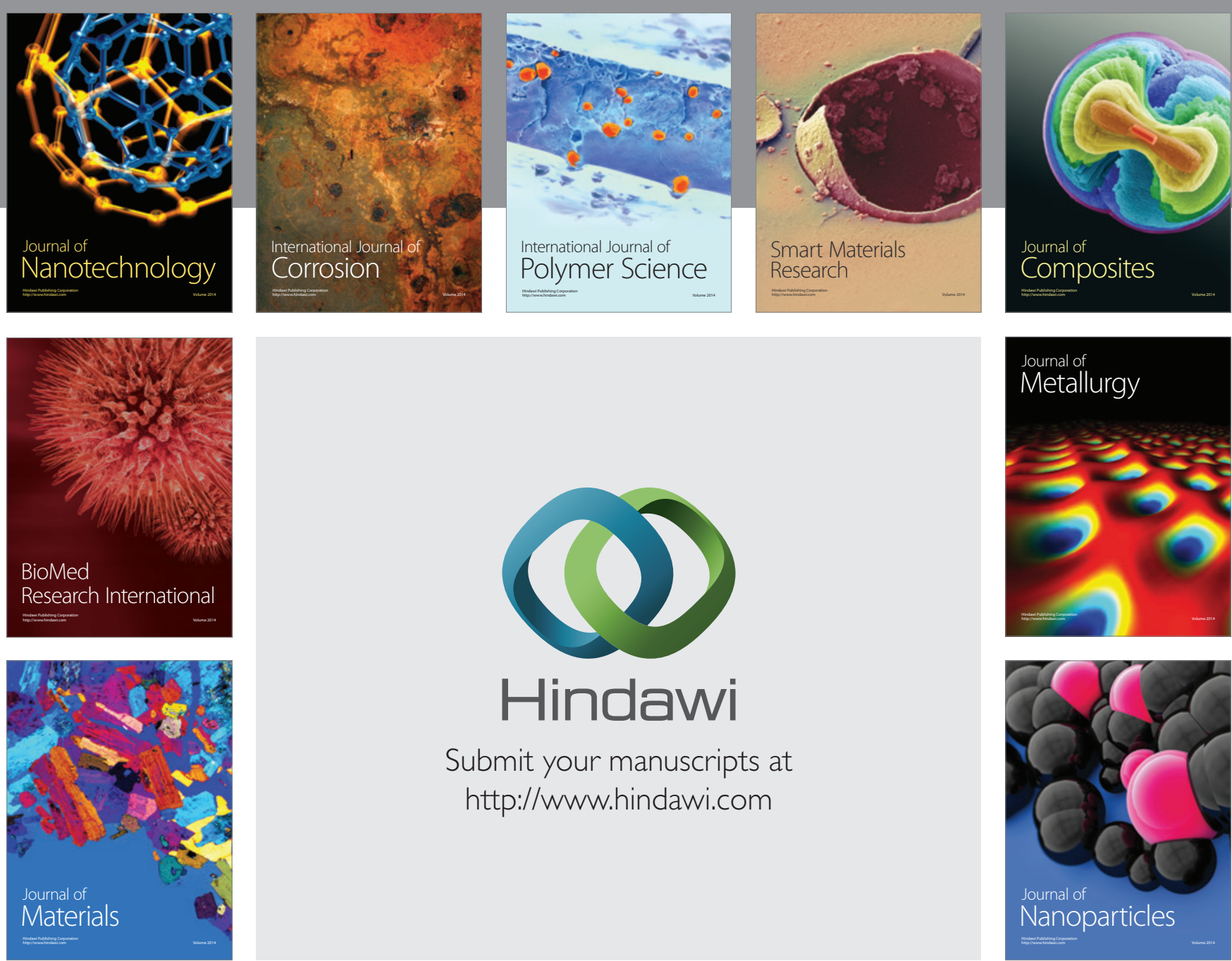

\section{Hindawi}

Submit your manuscripts at

http://www.hindawi.com

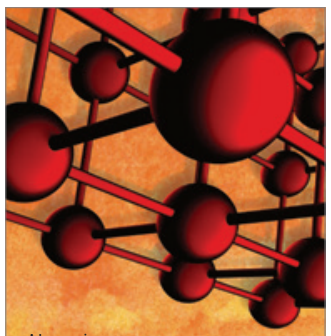

Materials Science and Engineering
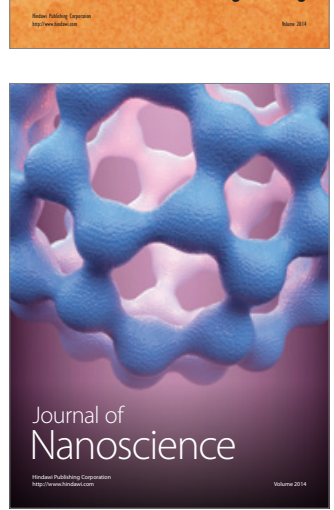
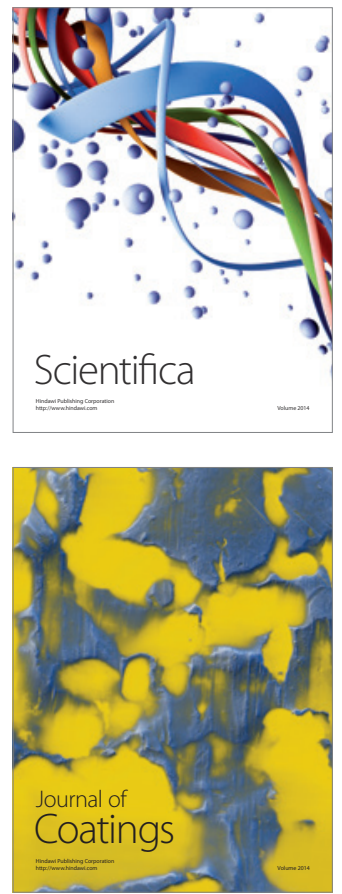
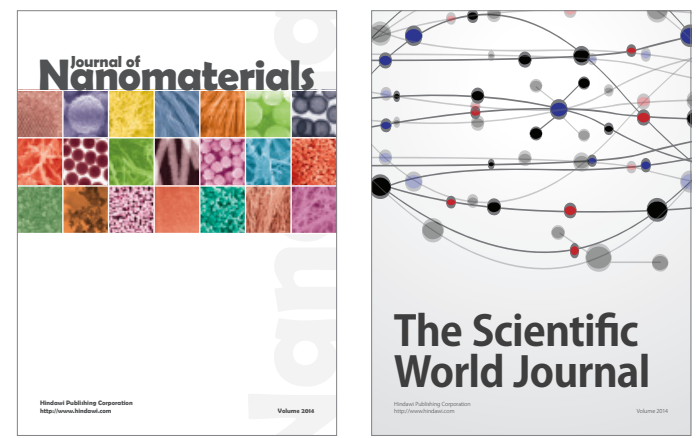

The Scientific World Journal
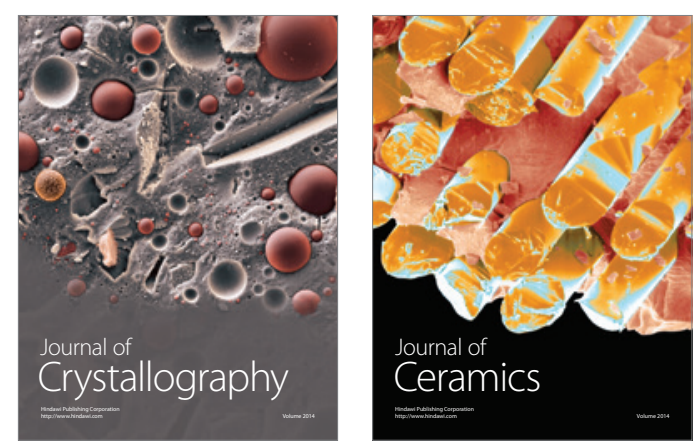
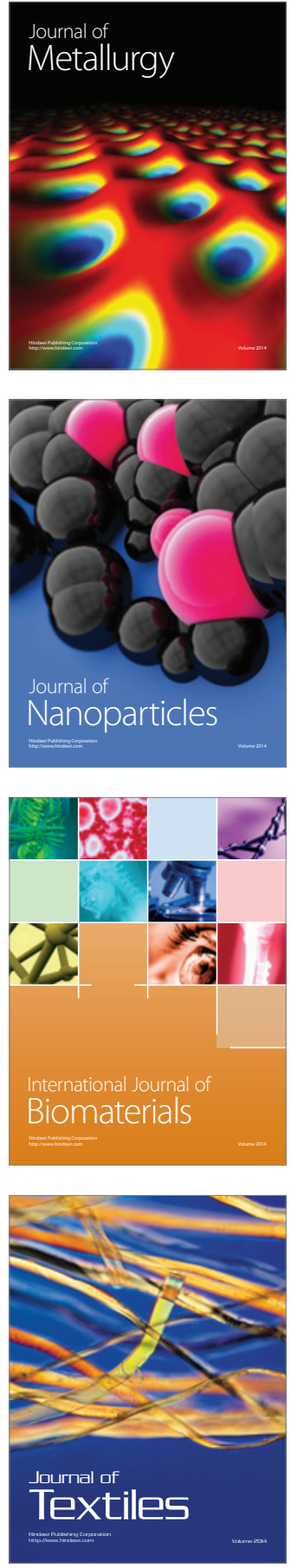